\title{
VNTR molecular typing of salmonella enterica serovar typhi isolates in Kathmandu valley
}

\author{
Acharya $\mathrm{B}^{1}$, Aryal $\mathrm{G}^{2}$, Banjade $\mathrm{N}^{4}$, Sharma $\mathrm{S}^{3}$, Pokharel $\mathrm{BM}^{3}$, Gotoh $\mathrm{A}^{1}$ \\ ${ }^{1}$ Laboratory of Cell and Gene Therapy Institute for Advanced Medical Sciences, Hyogo College of Medicine \\ Nishinomiya-shi, Hyogo, Japan \\ ${ }^{2}$ KIST Medical College and Teaching Hospital, Lalitpur, Nepal \\ ${ }^{3}$ Institute of Medicine, Tribhuwan University Teaching Hospital, Kathmandu, Nepal \\ ${ }^{4}$ Sidhhartha Polyclinic, Kathmandu, Nepal
}

\section{Keywords: \\ Salmonella enterica; \\ Variable number \\ Tandem Repeat; \\ Typhoid fever}

\begin{abstract}
Background: Typhoid fever continues to be a worldwide health problem, especially in developing countries. Effective epidemiological surveillance is needed to monitor the presence and spread of disease.

Materials and Methods: Variable number tandem repeats (VNTR) was performed for Salmonella enterica serovar typhi by multiplex-PCR in 28 Nepalese isolates of sporadic typhoid fever.

Results: From all 28 total isolates, we could identify 12 VNTR profiles among the isolates, signifying multiple variants in circulation within the region.

Conclusion: The VNTR-based typing assay for serovar typhi isolates can be used during an outbreak of enteric fever. The typing could eventually form the basis of an effective epidemiological surveillance system for developing rational strategies to control typhoid fever.
\end{abstract}

\section{INTRODUCTION}

There are an estimated 16 million typhoid fever cases with 600,000 related deaths world-wide. ${ }^{1}$ This highly adapted, human-specific pathogen has evolved remarkable mechanisms for persistence in its host that help to ensure its survival and transmission. The etiological agent, Salmonella(S) enterica serovar typhi, a gram-negative rodshaped bacterium is pathogenic only in humans, where it can be cultured from blood and stools. Infection occurs

\footnotetext{
Correspondence:

Akinobu Gotoh, MD, PhD

Laboratory of Cell and Gene Therapy Institute for Advanced Medical Sciences, Hyogo College of Medicine, 1-1 Mukogawa-cho, Nishinomiya-shi, Hyogo 663-8501, Japan.

Tel: +81-798-45-6808, Fax: +81-798-45-6806

E-mail: gotoh@hyo-med.ac.jp
}

when water or food contaminated with $S$. enterica serovar typhi is consumed. Most patients who recover from the infection are able to eliminate the bacterium completely from their bodies. However, some of them may remain as healthy carriers, continuously shedding $S$. enterica serovar typhi in their stools.

In Nepal, typhoid fever is endemic, severe in its manifestation as compared to patients from South American and African countries. There are limited reports of epidemics of typhoid fever in Nepal, which are usually attributed to the contamination of water source, especially during rainy season (June to August) when it floods. ${ }^{2}$ According to reports enteric fever (typhoid and paratyphoid fever) was the most common clinical diagnoses among febrile patients presenting to a general hospital in Kathmandu. ${ }^{3}$ It comprised 
sixty percent of clinically non-localizable fevers admitted to a regional Hospital. ${ }^{4}$

Epidemiological studies of pathogens are of great importance in controlling their dissemination. The capability to strain type pathogens is a critical tool in epidemiological investigations. Variable-number tandem repeats (VNTR) have been increasingly used as molecular makers for strain typing of various bacteria, including $S$. enterica serovar typhi. ${ }^{5}$ Conventional typing methods such as serotyping and phage typing have been and still are the mainstay in descriptive epidemiology of this microorganism. ${ }^{6}$ The resolving power of epidemiological typing has been expanded during recent years through the molecular analysis of microbial DNA using techniques such as pulsedfield gel electrophoresis (PFGE) and VNTR. Complete consensus has not yet been achieved on the techniques to use or the criteria for interpretation of the results, but these goals may be reached soon. We carried out the multiplexPCR in the isolates from Kathmandu valley in Nepal. The aim of the present study was to characterize serovar typhi strains isolated from the blood of enteric fever patients in Kathmandu, Nepal for the purpose of epidemiological surveillance and for better understanding of the disease pathogenecity in human.

\section{MATERIALS AND METHODS}

A total of 28 S. enterica serovar typhi isolates of sporadic cases collected over the year 2008 in a laboratory from the patients referred by the various private/public clinics in Kathmandu were included in this study. Strains of S. enterica serovar paratyphoid, were used as controls in this study. About 10 colonies from Lurie-Bertini agar medium were suspended in $500 \mu \mathrm{l}$ distilled water. The Cell suspension was boiled at $95{ }^{\circ} \mathrm{C}$ for 7 minute and then stored at $4{ }^{\circ} \mathrm{C}$ before being used directly for PCR.

The VNTR primers used for TR1 and TR2 loci were as described by Liu Et al. ${ }^{5}$

TR1 locus- Forward Primer 5'-AGA ACC AGC AAT GCG CCA ACG A-3

Reverse Primer 5 -CAA GAA GTG CGC ATA CTA CAC C-3

TR2 locus- Forward Primer 5 -CCC TGT TTT TCG TGC TGA TAC G-3'

Reverse Primer 5 '-CAG AGG ATA TCG CAA CAA TCG G-3’

Multiplex PCR reactions were performed in a volume of 20ul containing $1 \mathrm{ul}$ of the suspension of the boiled bacterial lysates, each deoxynucleotide triphosphate (dNTP) at $125 \mu \mathrm{mol} / \mathrm{L}, 2 \mathrm{U}$ of ExTaq polymerase, 1X Ex Taq Buffer, and 10 and $12.5 \mathrm{pmol}$ of TR1 and TR2 primers respectively. PCR was performed as follows:

Initial denaturation at $94{ }^{\circ} \mathrm{C}$ for 7 minutes, followed by 35

\begin{tabular}{|c|c|c|}
\hline Isolate reference \# & $\begin{array}{c}\text { VNTR profile } \\
\text { designation }\end{array}$ & $\begin{array}{c}\begin{array}{c}\text { Number of } \\
\text { isolates }\end{array} \\
\end{array}$ \\
\hline 27,56 & N1 & 2 \\
\hline $13,33,45,46,50(2)$ & N2 & 5 \\
\hline 19,24 & $\mathrm{~N} 3$ & 2 \\
\hline 30 & N4 & 1 \\
\hline $36,55,57,58$ & N5 & 4 \\
\hline $31,43,52$ & N6 & 3 \\
\hline $17,26,28,40$ & N7 & 4 \\
\hline $1,5,14$ & N8 & 3 \\
\hline 51 & N9 & 1 \\
\hline 42 & N10 & 1 \\
\hline 24 & N11 & 1 \\
\hline 79 & N12 & 1 \\
\hline Total & & 28 \\
\hline
\end{tabular}

cycles of melting at $94{ }^{\circ} \mathrm{C}$ for 30 seconds, annealing at 55 ${ }^{\circ} \mathrm{C}$ for 30 seconds, and extension at $72{ }^{\circ} \mathrm{C}$ for 45 seconds, with a final extension step of 7 minutes at $72{ }^{\circ} \mathrm{C}$ ( ASTEC PC-701 thermocycler).

PCR products were run on 3\% agarose gel at $100 \mathrm{~W}$ for 25 minutes, stained with ethidium bromide and detected with an ultraviolet ray camera.

BioMarker 0.1-12kb (Novagen) was applied to the same gel to determine the product size (Fig. 1).

\section{RESULTS}

Twenty eight isolates of $S$. enterica serovar typhi were analyzed. Amplicons of different sizes were observed for isolates when they were subjected to multiplex-PCR with primers for TR1 and TR2 (Fig.1). We arbitrarily named profiles N1-N12 for isolate strains. Profile N11 (then designated N1) was identified in a previous study. ${ }^{5}$ Profiles $\mathrm{N} 1$ to $\mathrm{N} 5$ are similar to the isolates from some countries of south Asia. However, N6-N12, which comprises majority of the isolates (Table 1), seems unique to Nepal. Repetition of the multiplex PCR confirmed that the results were reproducible. Multiplex PCR was performed on several $S$. enterica serovar enteritidis and $S$. enterica serovar Paratyphoid isolates for specificity of the assay. Two amplicons $\sim 200$ and 300 bp were observed for all reactions (Fig 1). This is in contrast to the highly polymorphic VNTR profiles in the $S$. enterica serovar typhi isolates.

\section{DISCUSSION}

The method of choice for typing S. serovar typhi as a means of source identification, outbreak investigation, and phylogenic studies is macrorestriction with PFGE 


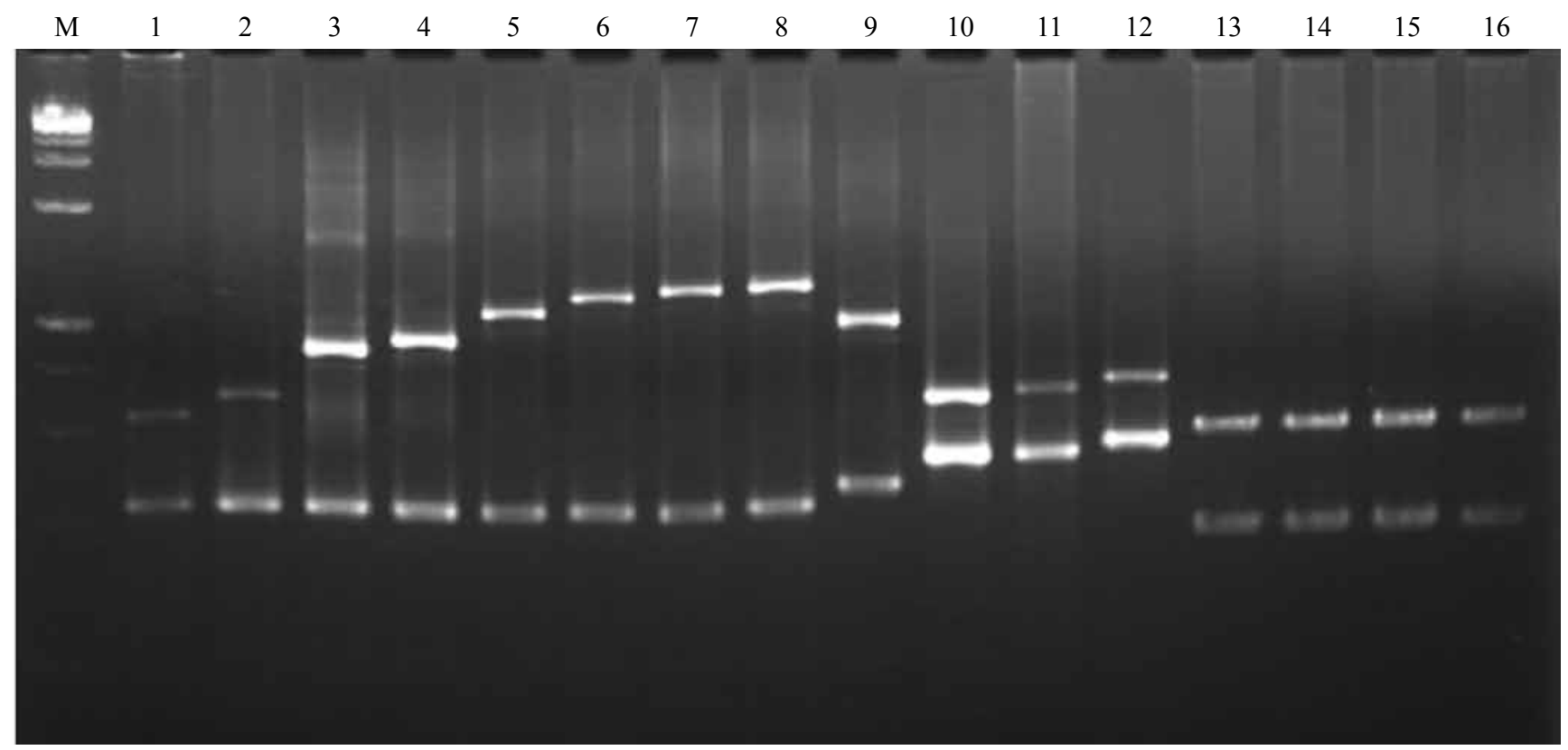

Figure 1: Agarose gel analysis of VNTR banding profiles amplified from 12 representative $S$. enterica serovar typhi isolates, S.enterica serovar Paratyphi and S. enterica serovar enteritidis by multiplex PCR containing TR1 and TR2 primer pairs. Lanes: 1 to 12, S. enterica serovar typhi isolates; 13-16, S.enterica serovar paratyphi. M, 100bp DNA marker.

separation of the fragments. PFGE has good discriminatory power, however, this method is quite labor-intensive, and the resulting electrophoretic patterns can be difficult to compare between different runs.

We utilized VNTR method for characterization of serovar typhi strains isolated from the blood of typhoid fever patients in Kathmandu valley. VNTR, or short sequence repeats, consist of unique DNA elements that are repeated in tandem. ${ }^{7}$ Individual strains within a bacterial species often maintain the same sequence element but with different copy numbers. Such variation is often caused by slipped-strand mispairing during DNA replication. ${ }^{8,9}$ Since sequence homology exists between strains in the flanking region of the VNTR locus, PCR amplification with flanking-sequencespecific primers can be used to determine the variations in copy numbers of repeat units that reflect the intraspecies genetic diversity. Therefore, individual strains can be easily identified by the amplicon sizes. This forms the basis for using VNTR for strain typing.

We found 12 VNTR types among 28 isolates. The banding profiles could be easily interpreted by visual inspection after electrophoresis on conventional agarose gels. We used only two loci TR1 and TR2 as it was determined that these were enough to discriminate between the isolates. The sizes of the amplicons did not change for 40 rounds of culture, suggesting that the loci have sufficient genetic stability for use as molecular size strain-typing markers. ${ }^{5}$ In addition, no changes in the sizes and sequences of TR1 and TR2 had been observed in the paired S. enterica serovar typhi isolates, specifically the three pairs that were collected from the same patients at different times. ${ }^{5}$ Our results have shown that this method is rapid, reproducible, and highly discriminative for the strain typing of $S$. enterica serovar typhi isolates

Substantial genetic heterogeneity at the VNTR loci exists among $S$. enterica serovar typhi isolates of Kathmandu valley. This finding is in accordance with previous reports ${ }^{5},{ }^{10-12}$ where genetic heterogeneity among and within geographical regions by using other conventional and molecular methods of typing was observed. VNTR typing in a limited number of Nepalese isolates (only 2) had been done by Liu et $\mathrm{al}^{5}$, where both strains were harboring identical VNTR types. High volume of human traffic could be the reason for genetic homogeneity among isolates. The cross-border traffic between Indian (free-boarder) and Bangladesh cities and Kathmandu is quite high. Therefore, it is reasonable to find several types in the isolate of Kathmandu. In addition patients in the study are from a wide geographical area comprising of near by provincial cities and villages where source of food and water is not the same. Hence, we can affirm that a multiple variants are circulating in the region.

The clinical importance of these differences remains to be fully evaluated. In this study it was not feasible to show a clear correlation between strain characteristics and disease severity. The clinical manifestations differ markedly in different parts of the world where typhoid is endemic. In South America and parts of Southeast Asia, typhoid fever manifests as a relatively mild illness with low fatality rates and minimal complications. In Nepal, severe and often fatal disease is frequently seen, with high mortality. The reasons for these differences in disease severity are not known but 
may be related to differences in health care facilities, host immune responses, genetic factors, and perhaps differences in the strains of $S$. enteric serovar typhi circulating in the area of endemicity.

\section{CONCLUSION}

The VNTR-based typing assay for serovar typhi isolates can be used during an outbreak of enteric fever. The typing could eventually form the basis of an effective epidemiological surveillance system for developing rational strategies to control typhoid fever.

\section{ACKNOWLEDGEMENTS}

We wish to thank Dr. Saleem Jahangeer for careful reading of the manuscript.

\section{REFERENCES}

1. Parry CM, Hien TT, Dougan G, White NJ, Farrar JJ. Typhoid fever. N Engl J Med 2002;347:1770-82.

2. Lewis MD, Serichantalergs O, Pitarangsi $\mathrm{C}$ et al. Typhoid fever: a massive, single-point source, multidrug-resistant outbreak in Nepal. Clin Infect Dis 2005;40:554-61.

3. Murdoch DR, Woods CW, Zimmerman MD et al. The etiology of febrile illness in adults presenting to Patan hospital in Kathmandu, Nepal. Am J Trop Med Hyg 2004;70:670-5.
4. Biswas R, Dhakal B, Das RN, Shetty KJ. Resolving diagnostic uncertainty in initially poorly localizable fevers: a prospective study. Int J Clin Pract 2004;58:26-8.

5. Liu Y, Lee MA, Ooi EE, Mavis Y, Tan AL, Quek HH. Molecular typing of Salmonella enterica serovar typhi isolates from various countries in Asia by a multiplex PCR assay on variable-number tandem repeats. J Clin Microbiol 2003;41:4388-94

6. Hampton MD, Ward LR, Rowe B, Threlfall EJ. Molecular fingerprinting of multidrug-resistant Salmonella enterica serotype Typhi. Emerg Infect Dis 1998;4:317-20.

7. Van Belkum A. The role of short sequence repeats in epidemiologic typing. Curr Opin Microbiol 1999;2:306-11.

8. Bzymek M, Lovett ST. Instability of repetitive DNA sequences: the role of replication in multiple mechanisms. Proc Natl Acad Sci USA 2001;98:8319-25.

9. Van Belkum A, Scherer S, van Alphen L, Verbrugh H. Shortsequence DNA repeats in prokaryotic genomes. Microbiol Mol Biol Rev 1998;62:275-93.

10. Franco A, Gonzalez C, Levine OS et al. Further consideration of the clonal nature of Salmonella typhi: evaluation of molecular and clinical characteristics of strains from Indonesia and Peru. J Clin Microbiol 1992;30:2187-90.

11. Ling JM, LO NW, HO YM et al. Molecular methods for the epidemiological typing of Salmonella enterica serotype Typhi from Hong Kong and Vietnam. J Clin Microbiol 2000;38:292-300.

12. Thong KL, Goh YL, Yasin RM et al. Increasing genetic diversity of Salmonella enterica serovar typhi isolates from papua new guinea over the period from 1992 to 1999. J Clin Microbiol 2002;40:415660 . 